Sel $f$-gener at $i$ on of hol I ow current prof $\mathrm{ile}$ and tilt instability in field-reversed conf $i$ gur at $i$ on

\begin{tabular}{|l|l|}
\hline $\begin{array}{l}\text { jour nal or } \\
\text { publ i cat i on } \mathrm{titl} \text { e }\end{array}$ & Physi cs of Pl asmas \\
\hline vol une & Vol . 10 \\
\hline number & I ssue1 \\
\hline page range & pp. 145- 156 \\
\hline year & 2003 10 - 01 \\
\hline URL & ht t p: //hdl . handl e. net /10655/3903 \\
\hline
\end{tabular}




\title{
Self-generation of hollow current profile and tilt instability in field-reversed configuration
}

\author{
Hiroaki Ohtania) \\ Theory and Computer Simulation Center, National Institute for Fusion Science, Toki 509-5292, Japan \\ Ritoku Horiuchi \\ Theory and Computer Simulation Center, National Institute for Fusion Science, Toki 509-5292, Japan \\ and Graduate University for Advanced Studies, Toki 509-5292, Japan \\ Tetsuya Sato \\ Earth Simulator Center, Japan Marine Science and Technology Center, Yokohama 236-0001, Japan
}

(Received 1 May 2002; accepted 10 October 2002)

\begin{abstract}
Two-dimensional electromagnetic particle simulation is performed to investigate the profile relaxation from a magnetohydrodynamic (MHD) equilibrium to a kinetic one and the physical property of the kinetic equilibrium in the field-reversed configuration. The radial oscillation is excited in order to relax an excess energy in the MHD equilibrium. After this profile oscillation, the system spontaneously relaxes toward a kinetic equilibrium, in which the electron current profile becomes hollow as a result of the combined effects of the gradient-B drift near the field-null line and the $\mathrm{E} \times \mathrm{B}$ drift generated by the ion finite Larmor radius effect near the magnetic separatrix. On the other hand, the ion current profile becomes peaked due to the effect of the ion meandering orbit near the field-null line. The stability of the obtained kinetic equilibrium against the tilt mode is also studied by means of three-dimensional full electromagnetic particle simulation. It is found that the growth rate of the tilt instability in the case of the hollow current profile and high separatrix beta value is smaller than that in the case of the peaked current profile. (C) 2003 American Institute of Physics. [DOI: 10.1063/1.1526703]
\end{abstract}

\section{INTRODUCTION}

The field-reversed configuration (FRC) has many advantages for a fusion reactor, as follows: (i) The geometry is very simple and compact with negligible toroidal field, in which a plasma is confined by poloidal magnetic field. (ii) The magnetic field is open in the periphery of plasma. (iii) The pressure of the plasma confined within the separatrix reaches its maximum value in the vicinity of the O-point, where there is no toroidal field and the magnetic field is reversed. Therefore, the averaged plasma beta $\langle\beta\rangle$ tends to be high in FRC plasmas $(\langle\beta\rangle \sim 1)$. The physics of FRC's have so far been studied from both theoretical and experimental points of view by many scientists. One of the most important studies is on the tilt instability.

The dangerous tilt instability in FRC plasmas, which leads to the disruption of plasma confinement, is predicted by magnetohydrodynamic (MHD) theory, ${ }^{1,2}$ but many experimental observations show that FRC plasmas remain stable much longer than the tilt growth time. ${ }^{3}$ Until now, various models with physical effects which are not taken in an ideal MHD theory have been studied in order to solve this contradiction. ${ }^{4-19}$ For example, Milroy et al. ${ }^{8}$ studied the nonlinear evolution of the tilt instability by using the threedimensional (3D) resistive MHD code with the Hall effect. Their numerical studies indicated that the Hall term does not suppress the tilt mode effectively in the range of the param-

${ }^{a)}$ Electronic mail: ohtani@tcsc.nifs.ac.jp eters used in most experiments. Barnes et al. ${ }^{4-7}$ evaluated numerically the finite ion Larmor radius (FLR) effect by using a Vlasov-fluid theory and found that the tilt mode can be stabilized for a kinetic plasma, but it tends to be unstable for a moderately kinetic plasma and the growth rate increases as the ion FLR effect decreases. Horiuchi and Sato ${ }^{9}$ executed the electromagnetic full particle simulation in which both ions and electrons were treated as particles and the initial condition was given by two-fluid equilibrium solution. Their simulation clarified that the tilt mode is stabilized for a kinetic plasma due to the effect of meandering ions which exist near the field-null line and have a large average velocity along the azimuthal direction. Many effects have been proposed as seen above, but none of them have given any satisfactory explanation as yet.

The tilt instability is also discussed from the viewpoint of the profile consistency. ${ }^{10,17-22}$ Steinhauer and Ishida ${ }^{20}$ analyzed the experimental measurements of several devices and pointed out that most experimental equilibrium configurations tend to take a hollow current profile. This analysis suggests that the hollow current profile may be relevant to the stabilization of the tilt mode. Cobb et al. ${ }^{10}$ clarified by making use of the extended MHD simulation with the Hall term that the equilibrium with hollow current profile is stable against the tilt mode if the beta value at the separatrix is enough large. In their model, however, the ion FLR effect increases as the current profile becomes hollow. Their result meant that the stable configuration is realized only in full kinetic plasmas, in which their model is not applicable. Be- 
lova et al. ${ }^{15,16}$ investigated the dependence of the global stability on the elongation numerically using both 3D MHD and hybrid (fluid electron and $\delta f$ particle ion) simulations. They found that the tilt mode growth rate for the prolate FRC is reduced due to the FLR effect, but complete linear stability is not found, even when FRC plasma is fully kinetic. Nishimura et al. ${ }^{11-14}$ revealed from the 3D electromagnetic full particle simulation that the tilt mode is stabilized for a large plasma beta value at the magnetic separatrix by the anchoring ions which play a role to hold the unstable internal plasma to the stable external plasma. The problem of their analysis is that the MHD equilibrium adopted as an initial condition relaxed to the kinetic one simultaneously with the evolution of the tilt instability and, therefore, the effect of the kinetic profile on the tilt mode is not clear.

Usually an MHD equilibrium is used for analysis of the tilt instability except for the cases in which the kinetic profile is dealt with under the restricted condition. ${ }^{10}$ It is important to obtain a kinetic profile of an FRC plasma and examine its character in considering the tilt instability because most experimental FRC plasmas are kinetic. It is quite difficult to give an analytic solution of a kinetic FRC equilibrium. However, we can clarify what kind of an equilibrium is realized in a kinetic FRC plasma, separately from the tilt mode by performing two-dimensional (2D) electromagnetic particle simulation because the tilt mode is $3 \mathrm{D}$ instability. In this paper, we first carry out the 2D electromagnetic particle simulation to sufficiently relax the system from an MHD equilibrium to a kinetic one separately from the tilt instability. From this simulation, the hollow current profile is found to be spontaneously generated through the relaxation process. In Sec. III, we examine the relaxation process of an FRC plasma from an initial MHD equilibrium to a kinetic one, and clarify its physical property. Section IV is devoted to discussing how the FRC plasma with the hollow current profile is kept stable against the tilt instability based on 3D full electromagnetic particle simulation in which the kinetic equilibrium obtained from the 2D simulation is used as the initial condition. Summary is given in Sec. V.

\section{SIMULATION MODEL}

In order to investigate the profile relaxation process independently of the tilt instability, we perform 2D simulation under the axisymmetry condition $(\partial / \partial \phi=0)$. The simulation starts from an MHD equilibrium. In this 2D simulation, we examine how the initial profile relaxes to a kinetic one and clarify what character a kinetic equilibrium has. Next we examine the stability against the tilt mode by performing $3 \mathrm{D}$ full electromagnetic particle simulation ${ }^{9,11-13}$ starting from the kinetic equilibrium obtained from the 2D simulation. Both 2D and 3D code relies on the semi-implicit method. ${ }^{23}$

The equations to be solved are the equations of motion

$$
\begin{aligned}
& \frac{d\left(\gamma_{j} \boldsymbol{v}_{j}\right)}{d t}=\frac{q_{j}}{m_{j}}\left(\boldsymbol{E}+\frac{\boldsymbol{v}_{j}}{c} \times \boldsymbol{B}\right), \\
& \frac{d \boldsymbol{x}_{j}}{d t}=\boldsymbol{v}_{j},
\end{aligned}
$$

and the Maxwell equations

$$
\begin{aligned}
& \frac{1}{c} \frac{\partial \boldsymbol{B}}{\partial t}=-\nabla \times \boldsymbol{E}, \\
& \frac{1}{c} \frac{\partial \boldsymbol{E}}{\partial t}=\boldsymbol{\nabla} \times \boldsymbol{B}-4 \pi \boldsymbol{j}, \\
& \boldsymbol{\nabla} \cdot \boldsymbol{B}=0, \\
& \boldsymbol{\nabla} \cdot \boldsymbol{E}=4 \pi \rho,
\end{aligned}
$$

where $\boldsymbol{x}_{j}(t), \boldsymbol{v}_{j}(t), m_{j}$, and $q_{j}$ are the position, the velocity, the rest mass and the charge of the $j$ th particle, and the relativistic $\gamma$-factor of the $j$ th particle is defined by

$$
\gamma_{j}=1 / \sqrt{1-\left(\boldsymbol{v}_{j} \cdot \boldsymbol{v}_{j}\right) / c^{2}} .
$$

The current density $\boldsymbol{j}(\boldsymbol{x}, t)$ and the charge density $\rho(\boldsymbol{x}, t)$ are obtained by summing over all the particles, namely

$$
\begin{aligned}
& \boldsymbol{j}(\boldsymbol{x}, t)=\sum_{j=1}^{N} \frac{q_{j} \boldsymbol{v}_{j}(t)}{c} S\left[\boldsymbol{x}-\boldsymbol{x}_{j}(t)\right], \\
& \boldsymbol{\rho}(\boldsymbol{x}, t)=\sum_{j=1}^{N} q_{j} S\left[\boldsymbol{x}-\boldsymbol{x}_{j}(t)\right],
\end{aligned}
$$

where $N$ is the total number of particles and $S(\boldsymbol{x})$ is the form function of particles which are expressed by a triangle with the base length equal to 2.0 times the grid separation. ${ }^{24,25}$

We consider an FRC plasma confined within a cylindrical conducting vessel by an external mirror magnetic field $\boldsymbol{B}_{\text {ex }}$ in order to control the shape of the separatrix. The external mirror magnetic field is obtained from the sum of two mirror potentials as

$$
\begin{aligned}
& \boldsymbol{B}_{\mathrm{ex}}=\left[-\frac{1}{r} \frac{\partial}{\partial z}\left(\Psi_{1}+\Psi_{2}\right), 0, \frac{1}{r} \frac{\partial}{\partial r}\left(\Psi_{1}+\Psi_{2}\right)\right], \\
& \Psi_{1}(r, z)=\Psi_{c 1} \frac{r}{r_{D}} \frac{J_{1}\left(k \frac{r}{z_{D}}\right)}{J_{1}\left(k \frac{r_{D}}{z_{D}}\right)} \cosh \left(k \frac{z}{z_{D}}\right), \\
& \Psi_{2}(r, z)=\Psi_{c 2} \frac{r}{r_{D}} \frac{I_{1}\left(k \frac{r}{z_{D}}\right)}{I_{1}\left(k \frac{r_{D}}{z_{D}}\right)} \cos \left(k \frac{z}{z_{D}}\right),
\end{aligned}
$$

where $J_{1}(x)$ and $I_{1}(x)$ are the Bessel and modified Bessel functions of first order, three constants $\Psi_{c 1}, \Psi_{c 2}$, and $k$ are the control parameters of the mirror field, and the half-height of this vessel $z_{D}$ is fixed to three times the vessel radius $r_{D}$ in this paper $\left(z_{D}=3 r_{D}\right)$. The control parameters of the mirror field we adopt in the simulation are summarized in Table I. It is assumed that the physical quantities are periodic at the boundary of the $z$ axis $\left(z= \pm z_{D}\right)$ and the vessel wall ( $r$ $=r_{D}$ ) is a rigid perfect conductor at which particles are elastically reflected. In 3D simulation, ${ }^{13}$ particle pusher and gather processes are carried out in the rectangular coordinates $(x, y, z)$ where the simulation domain is implemented on a $(49 \times 49 \times 32)$ point grid and its volume is given by 
TABLE I. The control parameters of the mirror field, $k$ and $\Psi_{c 2} / \Psi_{c 1}$. The kinetic parameter $\bar{s}$, the initial hollowness parameter $D$ and the mirror ratio are also shown. The cases from $\mathrm{C} 1$ to $\mathrm{C} 6$ is the results from the initial condition of the zero $E(0)$ case, and the cases from E1 to E6 is the results from the initial condition of the finite $E(0)$ case.

\begin{tabular}{ccccccc}
\hline \hline Case & C1,E1 & C2,E2 & C3,E3 & C4,E4 & C5,E5 & C6,E6 \\
\hline $\bar{s}$ & 1 & 1 & 2 & 2 & 3 & 3 \\
$D$ & -0.6 & 0.2 & -0.6 & 0.2 & -0.6 & 0.2 \\
$k$ & 0.75 & 0.75 & 0.75 & 0.75 & 0.75 & 0.75 \\
$\Psi_{c 2} / \Psi_{c 1}$ & 0.75 & 0.38 & 0.75 & 0.38 & 0.75 & 0.38 \\
mirror ratio & 0.827 & 1.00 & 0.827 & 1.00 & 0.827 & 1.00 \\
\hline \hline
\end{tabular}

$\left(2 r_{D} \times 2 r_{D} \times 2 z_{D}\right)$. On the other hand, the electromagnetic field is solved in the cylindrical coordinates $(r, \phi, z)$, and the $(33 \times 32 \times 32)$ space grids are used in this calculation. In $2 \mathrm{D}$ simulation, on the other hand, these processes are carried out under the axisymmetric condition. The total number of particles is $10^{6}$ in this simulation. The ratio of ion to electron mass $m_{i} / m_{e}$ is 50 and the frequency ratio $\omega_{p e} / \omega_{c e}$ is 5 , where $\omega_{p e}\left(=\sqrt{4 \pi n_{e} q_{e}^{2} / m_{e}}\right)$ is the electron plasma frequency defined by the density $n_{e}$ at the field-null line and $\omega_{c e}\left(=q_{e} B_{\text {wall }} / m_{e} c\right)$ is the electron cyclotron frequency defined by the magnetic field $B_{\text {wall }}$ at the vessel wall in the equatorial plane $(z=0)$. We adopt the predictor-corrector method with a sub-stepping of the electromagnetic field for a time advancing. Namely, the particles are advanced with a large time step of $\omega_{p e} \Delta t=1.5$, where $\Delta t$ is a time step, ${ }^{23}$ while the electromagnetic field is advanced with a small time step of $(c \Delta t) /(\Delta \phi \Delta r)<1.0$, where $\Delta \phi \Delta r$ is a minimum grid separation, ${ }^{26}$ by using an iteration method.

An initial profile of 2D simulation is obtained from the 2D one-fluid MHD equilibrium satisfying the GradShafranov equation

$$
-\nabla P+\frac{1}{c} \boldsymbol{J} \times \boldsymbol{B}=0 .
$$

The pressure function is given by the Cobb's pressure mode ${ }^{10}$ as

$$
P(\chi)=\left\{\begin{array}{c}
P_{0}\left(K_{0}-\chi-\frac{1}{2} D \chi^{2}\right) \\
\text { for } \chi \leqslant 0 ; \text { inside separatrix } \\
P_{0} K_{0} e^{-\chi / K_{0}} \\
\text { for } \chi \geqslant 0 ; \text { outside separatrix }
\end{array}\right.
$$

where $P\left(=P_{i}+P_{e}\right)$ is the total pressure, $\chi=\Psi /\left|\Psi_{\mathrm{ax}}\right|, \Psi_{\mathrm{ax}}$ is the value of $\Psi$ at the field-null $(\chi=-1)$, and $P_{0}$ is constant. $K_{0}$ is expressed by two important parameters as follows:

$$
K_{0}=\beta_{\mathrm{sp}} \frac{1-D / 2}{1-\beta_{\mathrm{sp}}},
$$

where $\beta_{\mathrm{sp}}$ is the normalized pressure value at the magnetic separatrix on the $z=0$ plane

$$
\beta_{\mathrm{sp}}=\frac{P(\chi=0)}{P(\chi=-1)}
$$

and also represents roughly the plasma beta value at the separatrix. $D$ is the hollowness parameter which controls the current distribution. That is to say, the current profile in the equilibrium state, $j_{\phi} / r$, becomes peaked for $D<0$, flat for $D=0$, and hollow for $D>0$.

It is very difficult to obtain a analytic profile in the kinetic plasma, such as a charge imbalance generated through the kinetic effect and a self-consistent profile of electric field with such charge imbalance. For simplicity, we choose two types of initial particle distribution to investigate the kinetic effects and the profile relaxation process from an MHD equilibrium to a kinetic one, because it is taken for granted that a kinetic equilibrium is quite different from an MHD one. The first one is the shifted-Maxwellian under the condition $E$ $=0$, where the average flow velocity is equal to the diamagnetic velocity and the ion temperature $\left(T_{i}\right)$ and the electron temperature $\left(T_{e}\right)$ are the same and spatially constant $\left(T_{i}\right.$ $=T_{e}=T$ ). This initial condition is more natural choice in order to examine the generation of $E$ and a charge imbalance in the simulation. The second one is the shifted-Maxwellian under the required $E \neq 0$, where the electric current is carried only by the electrons, the ion average flow velocity is zero, and $T_{i}=T_{e}=T$. The required $E$ is given by the ion fluid equation under zero ion mass flow velocity

$$
-\boldsymbol{\nabla} P_{i}+q_{i} n_{i} \boldsymbol{E}=0,
$$

where $n_{i}$ is the ion number density.

The parameter $\beta_{\mathrm{sp}}$ is fixed to 0.2 because the growth rate of the tilt instability is the smallest at this value in the simulations done by Nishimura et al. ${ }^{11}$ On the other hand, we adopt two types of current profile models, i.e., a peaked profile $(D=-0.6)$ and a hollow profile $(D=0.2)$, as a initial value for the MHD equilibrium in order to investigate the dependence of the kinetic equilibrium on the initial current profile and to examine whether the hollow profile is spontaneously realized in an FRC plasma.

We furthermore adopt the parameter $\bar{s}$ for the ion FLR effect as the another control parameter. The parameter $\bar{s}$ is defined by

$$
\bar{s}=\int_{R}^{r_{\mathrm{sp}}} \frac{r d r}{r_{\mathrm{sp}} \lambda_{i}},
$$

where $r_{\mathrm{sp}}$ is the separatrix radius, $R$ is the radius of the field-null line, and $\lambda_{i}$ is the local ion gyroradius at $z=0$. This parameter roughly indicates the ratio of the plasma radius to 


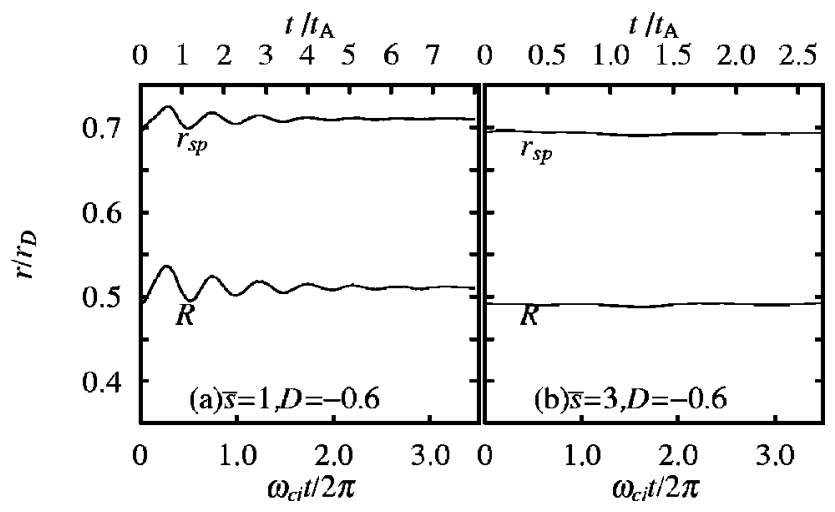

FIG. 1. Time evolution of the radii of the field-null line $R$ and separatrix $r_{\mathrm{sp}}$ for (a) $\bar{s}=1$ and (b) $\bar{s}=3 . r_{D}$ is the radius of the vessel, $\omega_{c i}$ is the ion gyration frequency, and $t_{\mathrm{A}}$ is the Alfvén transit time, respectively.

the ion Larmor radius. In this paper, we examine what kind of kinetic profile is realized and how the feature of the tilt mode is altered, when $\bar{s}$ varies from a full kinetic case $(\bar{s}$ $=1)$ to a moderate kinetic case $(\bar{s}=3)$.

\section{PROFILE RELAXATION}

In this section, we discuss the profile relaxation process from an MHD equilibrium to a kinetic one based on 2D simulation results.

\section{A. Relaxation from MHD to kinetic}

Let us examine how the plasma profile changes from an MHD equilibrium to a kinetic one under the initial condition where the average flow velocity $\langle v\rangle$ is equal to the diamagnetic velocity $v_{\text {dia }}$ and the initial electric field is zero $[E(0)$ $=0]$. Figure 1 shows the time evolution of the field-null line and separatrix radii $\left(R\right.$ and $\left.r_{\mathrm{sp}}\right)$ on the equatorial plane $(z$ $=0)$. In the full kinetic case $(\bar{s}=1)$ shown in Fig. 1(a), both $R$ and $r_{\mathrm{sp}}$ oscillate with the frequency $\omega \approx 2 \omega_{c i}$ in the early period $\left(\omega_{c i}\right.$ is the ion cyclotron frequency associated with the magnetic field at the conducting wall) and damp gradually until $\omega_{c i} t \approx 5 \pi$. This phenomenon indicates that the profile oscillates in the radial direction and that the system transits from the MHD equilibrium to a kinetic one during the relaxation period of $\omega_{c i} t=5 \pi$. Figure 2 shows the temporal evolution of the plasma beta value $\beta_{\mathrm{sp}}$ at the separatrix. In the case of $\bar{s}=1, \beta_{\text {sp }}$ jumps from an initial small value $\left(\beta_{\mathrm{sp}}=0.2\right)$ to about 0.3 in an initial moment $\left(\omega_{c i} t<\pi\right)$, and keeps this value after that. In the moderate kinetic case $(\bar{s}$ $=3$ ), on the other hand, no oscillation appears [Fig. 1(b)] and $\beta_{\text {sp }}$ keeps an initial value (Fig. 2). This radial oscillation takes place in order to relax an excess energy in the MHD equilibrium. When $\bar{s}$ is small or a plasma is fully kinetic, the energy difference between the initial MHD profile and the obtained kinetic equilibrium is so large that a relaxation oscillation is excited.

In order to examine the temporal behavior of the average current profile, we introduce the average hollowness parameter as

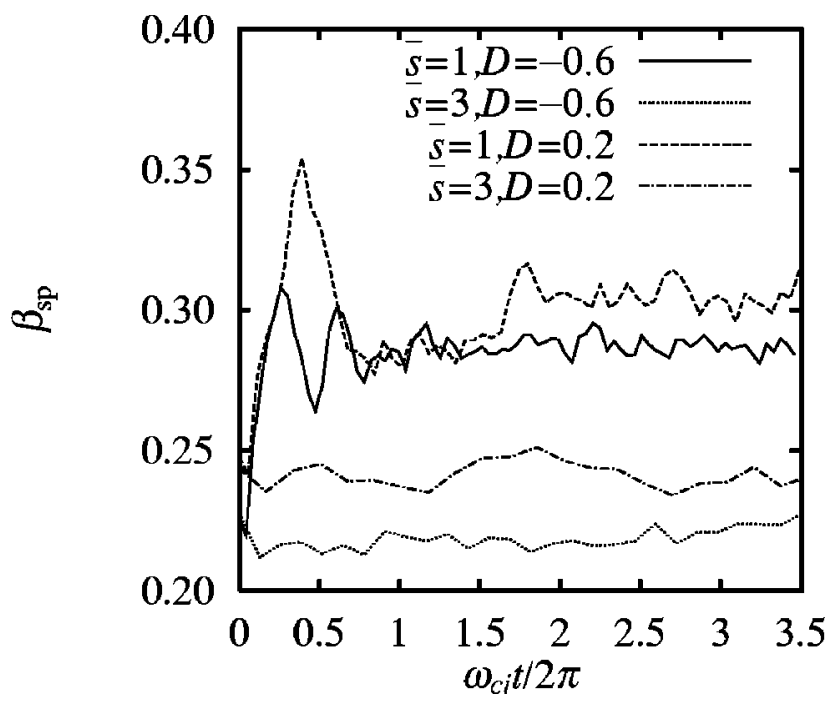

FIG. 2. Time evolution of the separatrix beta value $\beta_{\mathrm{sp}}$. The solid line shows the case of $\bar{s}=1$ and $D=-0.6$, the dotted line shows the case of $\bar{s}$ $=3$ and $D=-0.6$, the dashed-and-dotted line shows the case of $\bar{s}=1$ and $D=0.2$ and the broken line shows the case of $\bar{s}=3$ and $D=0.2$, respectively.

$$
\widetilde{D}(t)=\left.\left\langle-\frac{\left|\Psi_{a x}\right|}{c P_{0}} \frac{\partial}{\partial \chi}\left(\frac{J_{\phi}(t)}{r}\right)\right\rangle\right|_{R} ^{r_{\mathrm{sp}}},
$$

where $J_{\phi}(t)$ is the toroidal current density and $\left.\langle\cdots\rangle\right|_{R} ^{r_{\mathrm{sp}}}$ means the spatial average from $R$ to $r_{\mathrm{sp}}$. The parameter $D$ in an initial MHD profile corresponds to $\widetilde{D}(0)$ from Eqs. (13) and (14). Because the initial current is given by the diamagnetic current $\left(J_{e}=J_{\mathrm{dia}, e}, J_{i}=J_{\mathrm{dia}, i}\right)$ and the initial electron temperature is equal to the ion temperature $\left(T_{e}=T_{i}\right)$, the initial electron current density becomes equal to the ion current density $\left(J_{e}=J_{i}\right)$. Since the motion of the electron differs from that of the ion in a kinetic plasma, the electron current profile is expected to change from the ion one as time goes on. We, therefore, introduce the electron and ion hollowness parameters $\left(\widetilde{D}_{e}, \widetilde{D}_{i}\right)$ for the electron and ion current profile, respectively.

Figure 3 shows the time evolution of (a) the electron hollowness parameter $\widetilde{D}_{e}$ and (b) the ion one $\widetilde{D}_{i}$. The initial hollowness parameter $D$ is -0.6 for the peaked profile and 0.2 for the hollow one. Since the finite spatial mesh is used in the simulation, the observed value $\widetilde{D}(0)$ at $t=0$ is slightly different from the initial parameter $D$. One can see that the current profile changes immediately after the beginning of the simulation $\left(\omega_{c i} t \leqslant \pi\right)$. In the case of the initial peaked profile $(D<0)$ for the full kinetic case $(\bar{s}=1), \widetilde{D}_{e}$ shifts from negative value to positive one. This result indicates that the electron current profile is changed from the peaked one to the hollow one. The absolute value of $\widetilde{D}_{i}(<0)$ increases, that is, the ion current profile becomes more peaked. In the case of the initial hollow profile $(D>0)$ for full kinetic case $(\bar{s}=1)$, on the other hand, the absolute value of $\widetilde{D}_{e}$ increases, that is to say, the electron current profile becomes more hollow. $\widetilde{D}_{i}$ changes from positive value to negative one, namely the ion current profile is changed from the hol- 


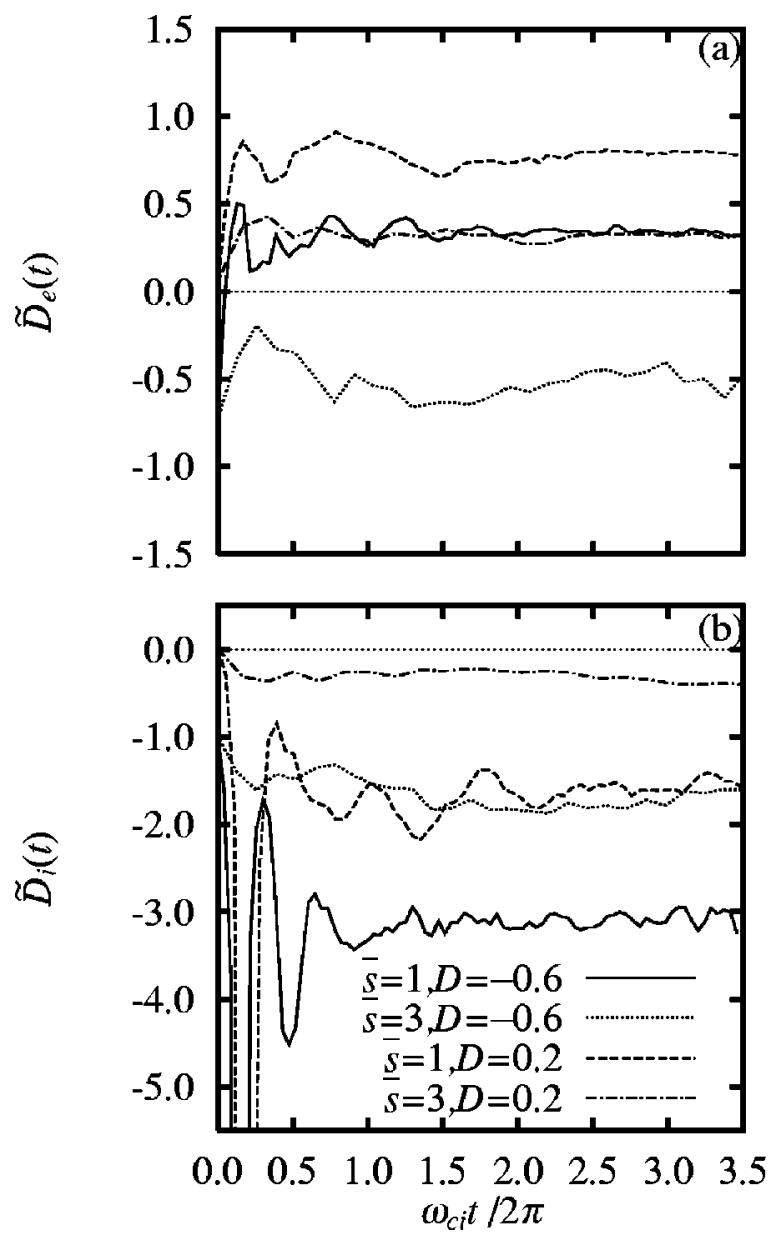

FIG. 3. Time evolution of the hollowness parameter for (a) the electron current density $\widetilde{D}_{e}(t)$ and (b) the ion current density $\widetilde{D}_{i}(t)$. The solid line shows $\bar{s}=1$ and $D=-0.6$, the dotted line shows $\bar{s}=3$ and $D=-0.6$, the dashed-and-dotted line shows $\bar{s}=1$ and $D=0.2$ and the broken line shows $\bar{s}=3$ and $D=0.2$, respectively.

low one to the peaked one. When the FRC plasma is moderately kinetic $(\bar{s}=3), \widetilde{D}_{e}$ and $\widetilde{D}_{i}$ almost keep the initial value both in the cases of $D=-0.6$ and 0.2. On the average, the electron current profile changes to a hollow one and the ion current profile changes to a peaked one independently of the initial current profile as a result of the profile relaxation.

\section{B. Current profile in a kinetic equilibrium}

In the previous subsection, we find that the electron and ion current profiles change to hollow and peaked ones after the relaxation, respectively. In this subsection, we first examine in detail how the spatial profile of the current density changes when the MHD equilibrium relaxes to the kinetic one $\left(\omega_{c i} t>5 \pi\right)$. Figure 4 shows the spatial profile of the toroidal current density $J_{\phi}$ for $\bar{s}=1$ and $D=-0.6$ at (a) $\omega_{c i} t=0$ and (b) $\omega_{c i} t=5 \pi$, which is averaged in terms of the $\phi$ variation. The electron current density $J_{\phi, e}$ decreases near the field-null line, and increases near the separatrix. Totally, an initial peaked profile changes to a hollow profile. On the other hand, the ion current density $J_{\phi, i}$ increases near the field-null line, and then the ion current profile becomes more peaked. So the total current density $J_{\phi, t}$ near the field-null
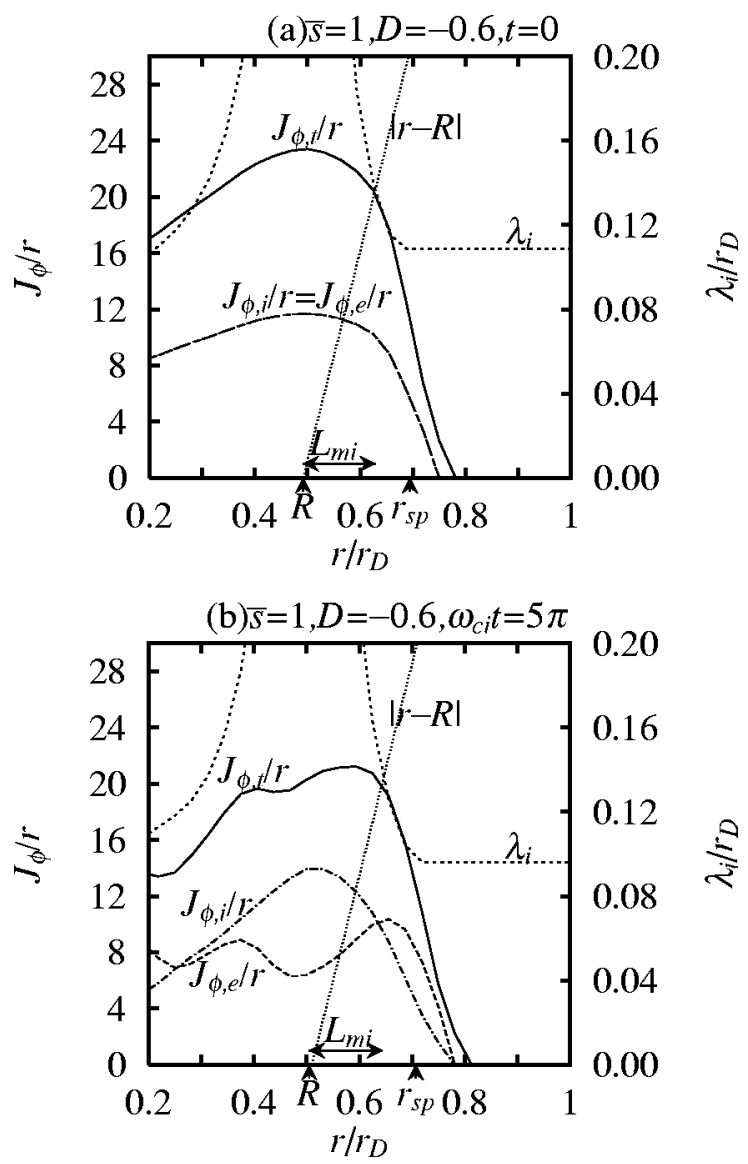

FIG. 4. Radial profile of the toroidal current density for the case of $\bar{s}=1$ and $D=-0.6$ at (a) $t=0$ and (b) $\omega_{c i} t=5 \pi$, which is averaged in terms of the $\phi$ variation. The solid line shows the total current density $J_{\phi, t} / r$, the broken line shows the electron current density $J_{\phi, e} / r$, the dashed-and-dotted line shows the ion current density $J_{\phi, i} / r$, the dashed line shows the local ion Larmor radius $\lambda_{i}(r)$, and the dotted line shows the distance from the fieldnull line $|r-R|$, respectively. $L_{m i}$ is the ion meandering orbit amplitude.

line decreases and then its profile changes from the initial peaked one to the hollow one. The spatial structure of the electron toroidal current density $J_{\phi, e}$ is shown in Fig. 5 for the case of $\bar{s}=1$ and $D=-0.6$ at the initial time $(t=0)$ and after the relaxation $\left(\omega_{c i} t=5 \pi\right)$ in the $r-z$ plane. It is clearly seen in Fig. 5 that though the electron current profile is peaked around the field-null line at the initial time, it changes to the hollow profile around there after profile relaxation.

Next we compare this current profile change in $\bar{s}=1$ with that in $\bar{s}=3$ (a moderate kinetic case) to examine its dependence on the ion FLR parameter $\bar{s}$. Figure 6 is the same figure as Fig. 4 but for $\bar{s}=3$ and $D=-0.6$. In the case of $\bar{s}=3$, the electron current profile becomes slightly hollow and the ion current profile becomes slightly peaked. However, they do not change so much as in the case of $\bar{s}=1$. Let us next examine the dependence on the initial hollowness parameter $D$. Figure 7 is the same figure as Fig. 4 but for $D=0.2$ and $\bar{s}=1$. In the case of $\bar{s}=1$ and $D=0.2, J_{\phi, i}$ increases and $J_{\phi, e}$ decreases near the field-null line, that is, the ion current profile changes from the initial hollow one to the peaked one and the electron current profile becomes more hollow. The total current profile also becomes more hollow. 


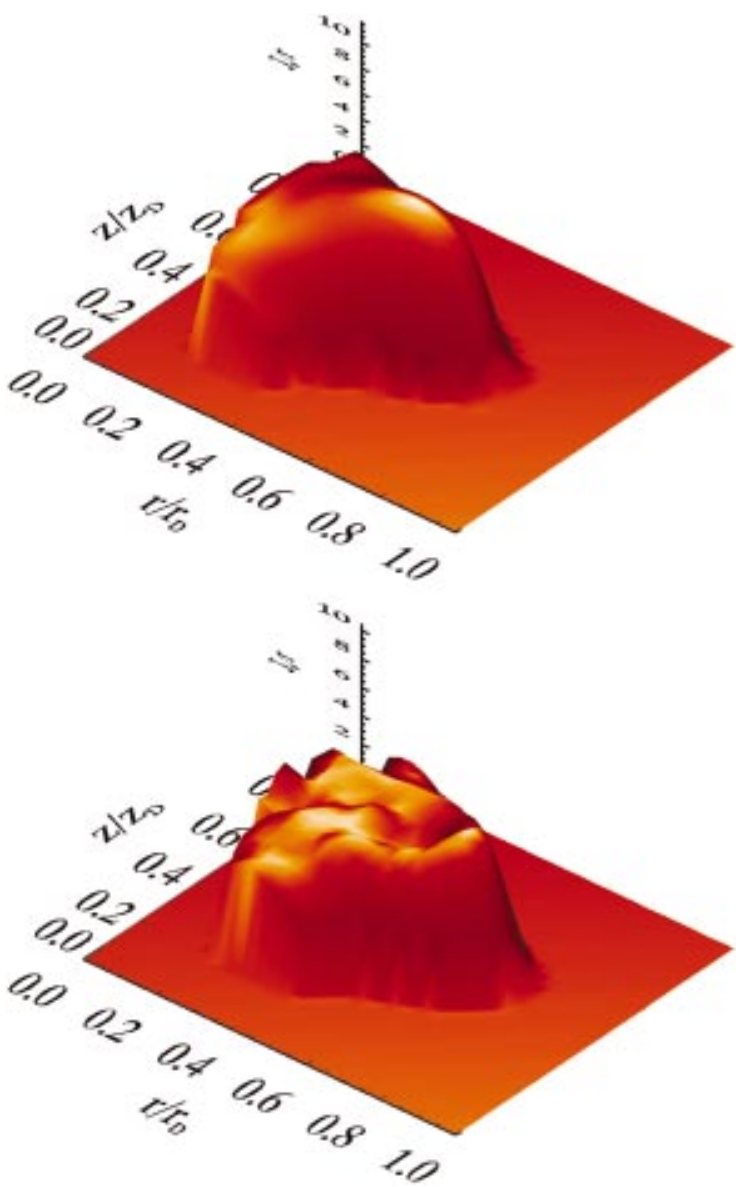

FIG. 5. (Color) The perspective diagrams of the electron toroidal current density $J_{\phi, e} / r$ for the case of $\bar{s}=1$ and $D=-0.6$ at $t=0$ (top) and $\omega_{c i} t$ $=5 \pi$ (bottom). Here, the vertical axis shows $J_{\phi, e} / r$ in the $r-z$ plane, which is averaged in terms of the $\phi$ variation.

The more hollow profile is realized in this case compared with in $\bar{s}=1$ and $D=-0.6$ [Fig. 4(b)].

Consequently, in the full kinetic case, $J_{\phi, i}$ increases and $J_{\phi, e}$ decreases near the field-null line, while $J_{\phi, e}$ increases near the separatrix independently of the initial hollowness value $D$. In the moderate kinetic case, on the other hand, their behaviors show the same tendency as in $\bar{s}=1$, though their changes are relatively small. One can see that how the hollow profile of the total current is created near the fieldnull line dependently on the values of $\bar{s}$ and $D$. This interesting simulation result that the current profile spontaneously changes to a hollow one is in good agreement with the analysis of experimental results. ${ }^{20}$

Let us consider the reasons why the electron current profile becomes hollow and the ion current profile becomes peaked, that is to say, what causes the plasma current to shift from the initial diamagnetic profile. We separately discuss the reasons near the field-null line and the separatrix since the different tendency is observed there.

\section{Near the field-null line}

The single particle orbit is an important keyword in considering the reasons why the electron current profile becomes hollow and the ion current profile becomes peaked near the field-null line, respectively.
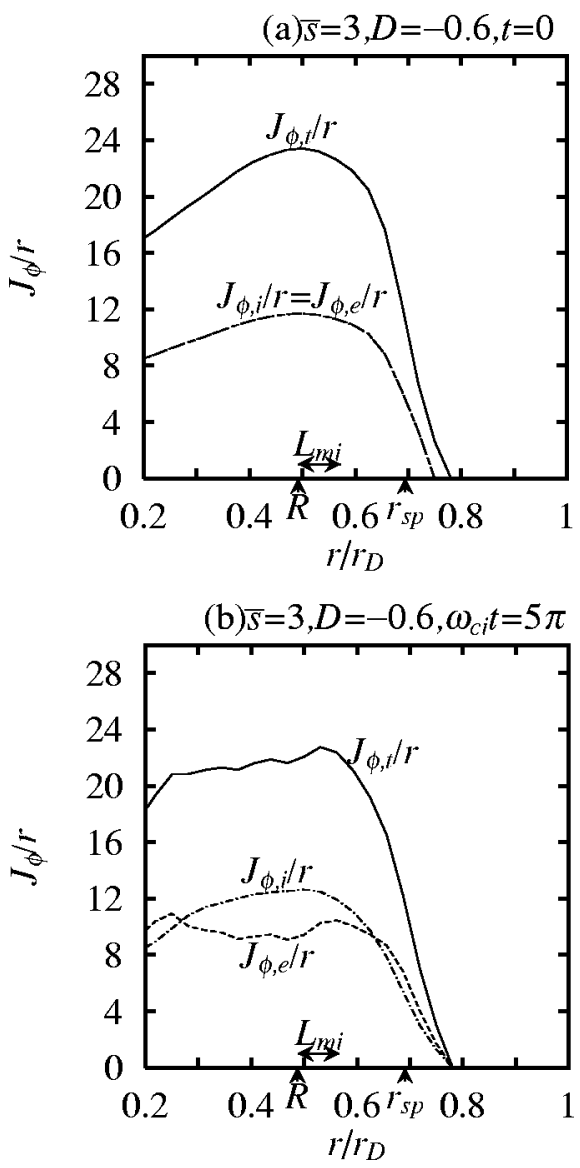

FIG. 6. The same figure as Fig. 4 but for the case of $\bar{s}=3$ and $D=-0.6$.

Figure 8 shows the spatial profiles of the various electron velocities along the toroidal direction on the equatorial plane $(z=0)$ for $\bar{s}=1$ and $D=-0.6$ at (a) $\omega_{c i} t=0$ and (b) $\omega_{c i} t=5 \pi$. The average flow velocity (solid) is equal to the diamagnetic velocity (dotted) at $\omega_{c i} t=0$. It is clear in Fig. 8 that the dominant electron motion on the equatorial plane $(z=0)$ is the gradient-B drift near the field-null line. Therefore, the electron current near the field-null line is roughly given by the sum of the diamagnetic current and the gradient-B drift current $\left(J_{e} \sim J_{\mathrm{dia}, e}+J_{\nabla B}\right)$. Because the gradient-B drift has the opposite sign to the electron diamagnetic drift [Fig. 8(b)], the current density decreases near the field-null line. Strictly speaking, the drift orbit approximation is not applicable to the vicinity of the field-null line $(\mid r$ $-R \mid<\lambda_{e}(r), \lambda_{e}(r)$ : The local electron Larmor radius), because the electron Larmor radius becomes infinite. But, the tendency is correct.

When the spatial scale of the magnetic field is almost the same as ion orbit scale $(\bar{s}=1)$, ions execute meandering motions along the field-null line without any self-intersection of the orbit line. ${ }^{9}$ The spatial size $L_{m i}$ of this meandering motion is determined from the location which satisfies the relation

$$
L_{m i} \equiv \lambda_{i}(r)=|r-R|,
$$

where $\lambda_{i}(r)$ is the local ion Larmor radius (see Fig. 4). For comparison, we plot the ion meandering orbit amplitude $L_{m i}$ in Figs. 4, 6, and 7. When the FRC plasma is fully kinetic, or 

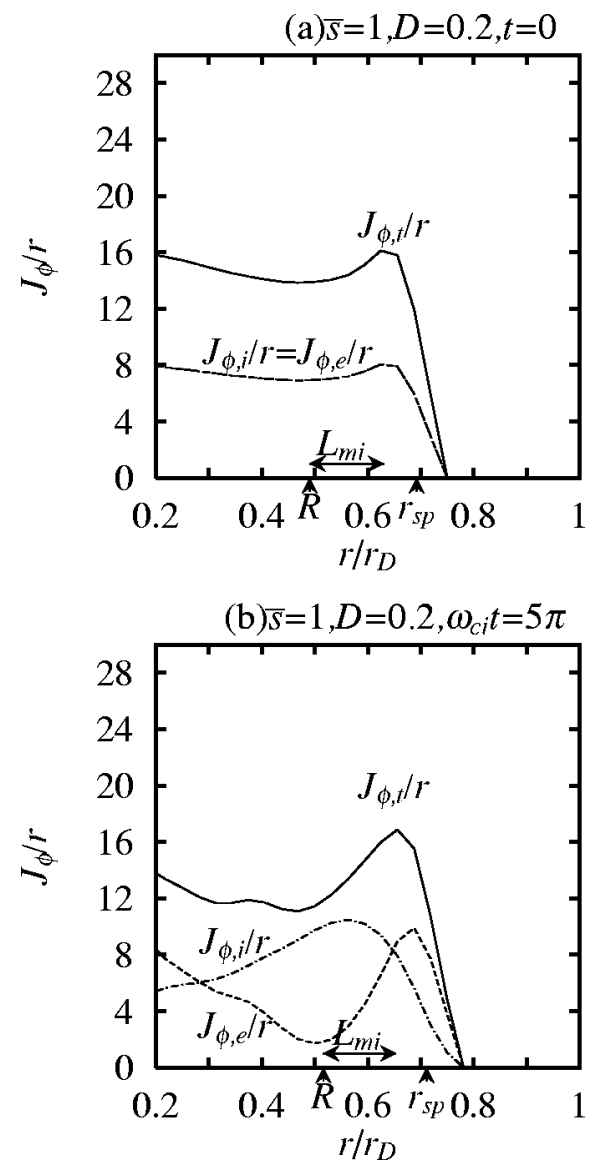

FIG. 7. The same figure as Fig. 4 but for the case of $\bar{s}=1$ and $D=0.2$.

$\bar{s}$ is small, $L_{m i}$ is large and the average toroidal velocity is also large due to this meandering motion. Accordingly, the large average toroidal velocity causes $J_{\phi, i}$ to increase near the field-null line.

To confirm that both the decrease of $J_{\phi, e}$ and the increase of $J_{\phi, i}$ near the null line can be explained by the character of the single particle orbit, we calculate the orbits of particles, which satisfy the same initial conditions as the 2D simulation, under the fixed field. Figure 9 shows the time evolution of the spatial profile of the current densities $J_{\phi, e}$ and $J_{\phi, i}$ obtained from this orbit calculation in the case of $\bar{s}=1$ and $D=-0.6$. Due to only the effect of the single particle orbit, the re-distribution of the momentum takes place in space, and $J_{\phi, i}$ increases and $J_{\phi, e}$ decreases near the field-null line, respectively.

Note that both $J_{\phi, e}$ and $J_{\phi, i}$ do not change near the separatrix. This tendency for the electron current to become hollow and for the ion current to become peaking near the fieldnull line is the same as the results of $2 \mathrm{D}$ simulation [Fig. 4(b)]. It is confirmed that $J_{\phi, e}$ decreases and $J_{\phi, i}$ increases near the field-null line because of the effect of the single particle orbit.

\section{Near the separatrix}

Because the density profile becomes steep locally in the narrow periphery region near the separatrix, the ion finite Larmor radius effect generates the radial electric field $E_{r}$

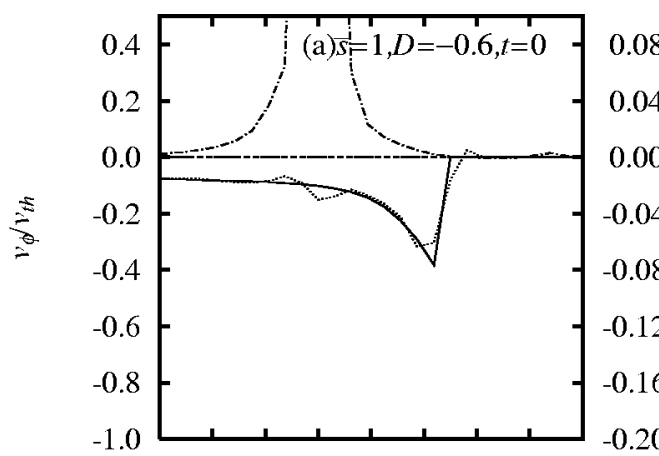

$\Delta$

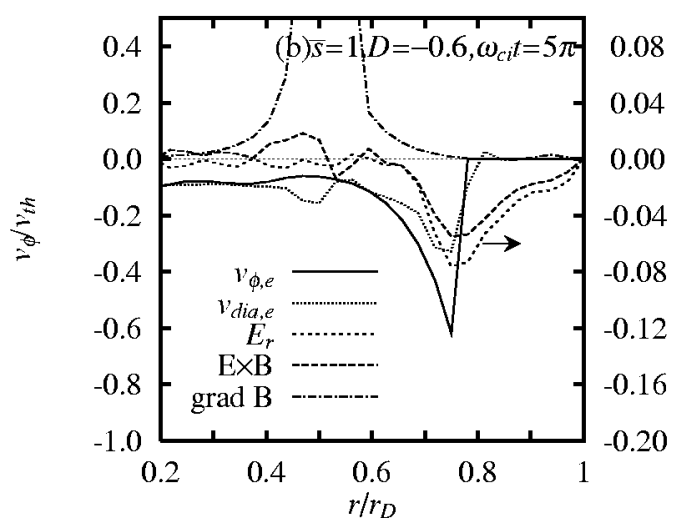

FIG. 8. Electron average toroidal velocity at (a) $t=0$ and (b) $\omega_{c i} t=5 \pi$. The solid line shows the electron velocity $v_{\phi, e}$, the dotted line shows the electron diamagnetic velocity $v_{\mathrm{dia}, e}$, the dashed line shows the radial electric field $E_{r}$, the broken line shows the $\mathrm{E} \times \mathrm{B} \operatorname{drift}(E \times B)$, and the dashed-anddotted line shows the gradient-B drift ( $\operatorname{grad} B)$, respectively.

there (Fig. 8). Since the generated $E \times B$ drift there has the same sign as the electron diamagnetic drift, $J_{\phi, e}$ increases in the periphery. Though the $E \times B$ drift has the opposite sign as the ion diamagnetic drift, the spatial size of the strong electric field region is smaller than the ion Larmor radius and so $E_{r}$ acts on ions less effectively. Thus the modification of the ion current profile becomes relatively smaller and $J_{\phi, i}$ slightly decreases near the separatrix.

In this way, the electron current density decreases and the ion current density increases near the field-null line,

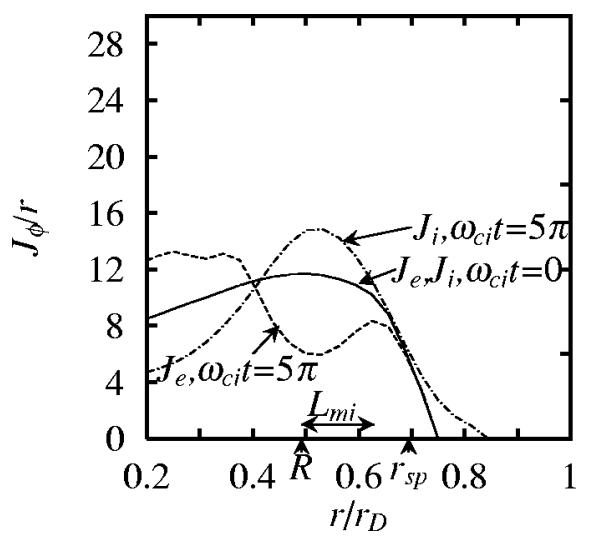

FIG. 9. Radial profile of the toroidal current density in the orbit calculation for the case of $\bar{s}=1$ and $D=-0.6$. The solid line show the current at $t$ $=0$, the broken and dashed-and-dotted lines show the electron and ion current at $\omega_{c i} t=5 \pi$, respectively. $L_{m i}$ is the ion meandering orbit amplitude. 

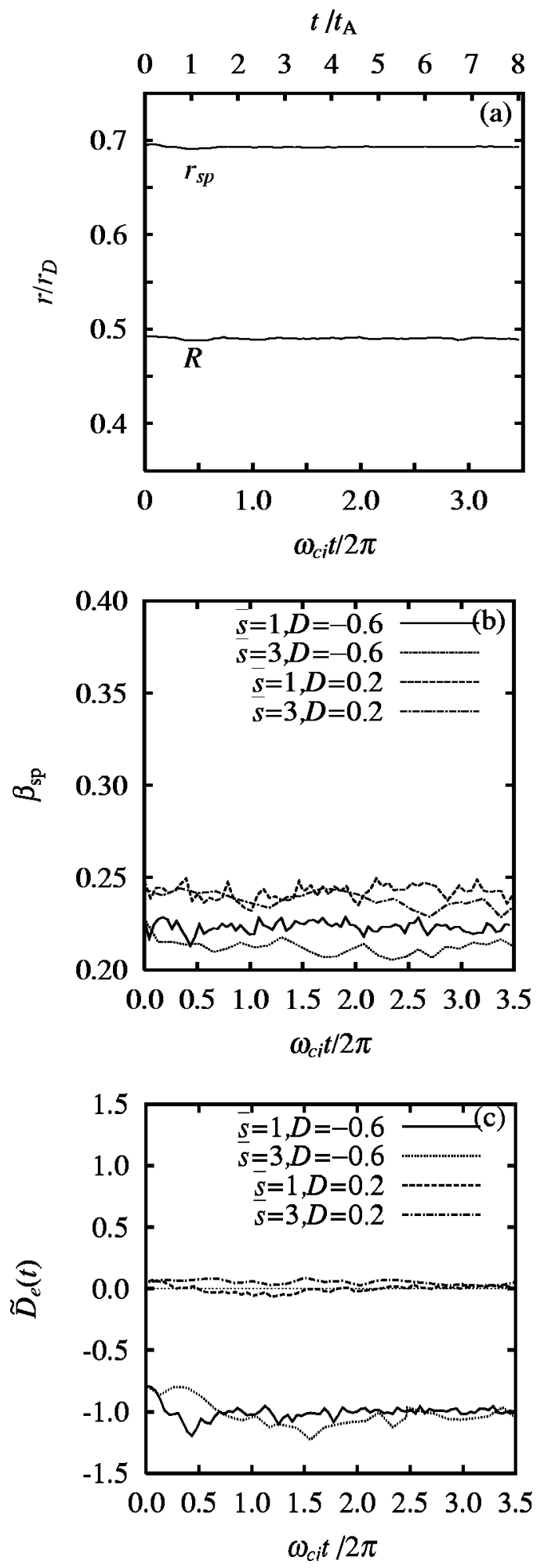

FIG. 10. The temporal evolutions of (a) $R$ and $r_{\text {sp }}$, (b) $\beta_{s p}$, and (c) $\widetilde{D}_{e}$ in the initial condition of the finite $E(0)$ case.

while the electron current density increases near the separatrix. Accordingly, an initial MHD equilibrium relaxes to a kinetic equilibrium with the electron hollow and ion peaked current profiles through the combined effects of the single particle orbit and ion finite Larmor radius.

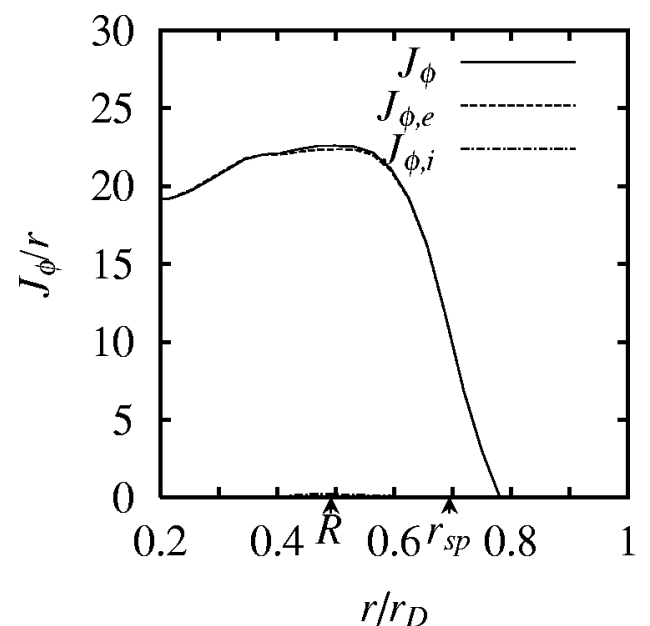

FIG. 11. The same figure as Fig. 4 but for the case of $\bar{s}=1$ and $D=-0.6$ in the initial condition of the finite $E(0)$ case at $\omega_{c i} t=5 \pi$.

\section{Effects of ion mass flow}

So far we discuss the results when the simulation starts from the MHD equilibrium with the average ion flow velocity equals to the diamagnetic one. The effects of ion mass flow is not negligible because the average ion flow velocity is comparable to the thermal ion velocity $\left(\left\langle v_{i}\right\rangle \sim 0.4 v_{T i}\right)$ in the full kinetic case of $\bar{s}=1$. So let us compare the results from the initial condition, where the electric current is carried only by the electrons, the average ion flow velocity $\left\langle v_{i}\right\rangle$ is zero, in the finite $E(0)$ case, with the results from the initial condition, where $\langle v\rangle$ is equal to $v_{\mathrm{dia}}$ in the zero $E(0)$ case.

Figure 10 show the temporal evolutions of (a) $R$ and $r_{\mathrm{sp}}$, (b) $\beta_{\mathrm{sp}}$ and (c) $\widetilde{D}_{e}$ in the finite $E(0)$ case. In the zero $E(0)$ case, the profile oscillation takes place in the radial direction (see Fig. 1), $\beta_{\mathrm{sp}}$ increases from an initial value (see Fig. 2), and $\widetilde{D}_{e}$ becomes positive value (see Fig. 3 ), while in the finite $E(0)$ case, $R, r_{\mathrm{sp}}, \beta_{\mathrm{sp}}$ and $\widetilde{D}_{e}$ keep almost an initial values. This results indicates that the system transits from the MHD equilibrium to a kinetic one without any clear profile oscillation in the finite $E(0)$ case. The reason for this difference is that the centrifugal force by kinetic effect exerts an influence on ions when the system has a finite ion flow in the full kinetic case. When the initial MHD profile has the ion average flow velocity which equals to the diamagnetic one, it does not include the centrifugal effects and then the relaxation oscillation is excited to relax the energy difference between the initial MHD profile and the obtained kinetic equilibrium.

Figure 11 shows the spatial profile of $J_{\phi}$ for $\bar{s}=1$ and $D=-0.6$ at $\omega_{c i} t=5 \pi$ in the finite $E(0)$ case. After relaxation, $J_{\phi, i}$ is generated through the meandering effect. However, its magnitude is small because the initial ion current is zero. On the other hand, $J_{\phi, e}$ slightly decreases near the field-null line due to the gradient- $B$ drift and increases near the separatrix because of the $E \times B$ drift, and then the electron current profile merely changes to the hollow profile around the field-null line. The reason for this slightly decrease of $J_{\phi, e}$ is that the radial electric field also exists 


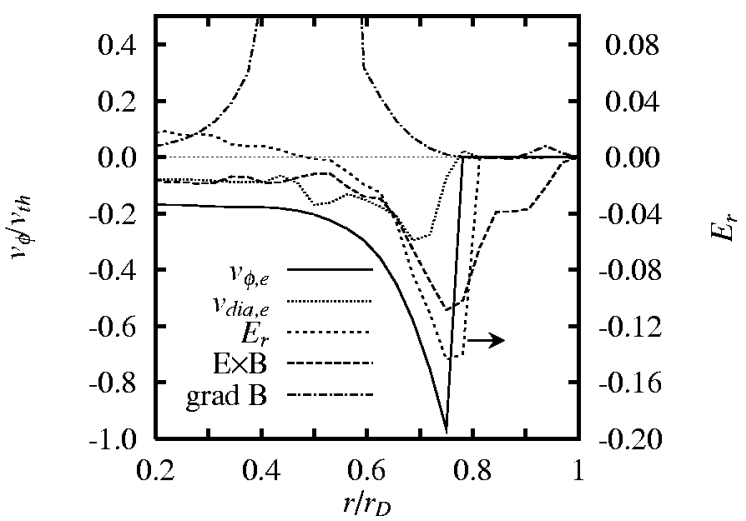

FIG. 12. The same figure as Fig. 8 but for the case of $\bar{s}=1$ and $D=-0.6$ in the initial condition of the finite $E(0)$ case at $\omega_{c i} t=5 \pi$.

around the null-line shown in Fig. 12 and then $E \times B$ drift is generated there. Because this generated $E \times B$ has the same sign as the electron diamagnetic drift, $J_{\phi, e}$ slightly decreases there though the gradient-B drift reduces $J_{\phi, e}$. It is also confirmed from the orbit calculation in the fixed electromagnetic field that the initial current profile is kept without any significant change in the presence of the finite $E(0)$ field.

Consequently, the difference between the kinetic equilibrium and the MHD one under the initial condition where $\langle v\rangle$ is equal to $v_{\text {dia }}$ and $E(0)=0$ is large in the full kinetic case and it is small in the moderate kinetic (MHD-like) case. On the other hand, under the initial condition where the electric current is carried only by the electrons, $\left\langle v_{i}\right\rangle$ is zero, and $E(0) \neq 0$, it is not so large both in the full kinetic and moderate cases. In this way, we obtain several kinetic equilibrium configurations from several initial conditions.

\section{TILT INSTABILITY}

In the previous section, we find from 2D simulation that the kinetic equilibrium with the hollow current profile is spontaneously generated in the FRC plasma. In this section, we examine the stability against the tilt mode in the kinetic equilibrium based on 3D simulation results. In the 3D simu- lation, the kinetic equilibrium solution obtained at $\omega_{c i} t$ $=7 \pi$ (after the profile relaxation) in $2 \mathrm{D}$ simulation is adopted as the initial condition.

The time averaged parameters, the field-null and separatrix radii $\left(\langle R\rangle\right.$ and $\left.\left\langle r_{\mathrm{sp}}\right\rangle\right)$, the elongation $\left(\left\langle E\left(=z_{\mathrm{sp}} / r_{\mathrm{sp}}\right)\right\rangle\right)$, the plasma beta value at the separatrix $\left(\left\langle\beta_{\mathrm{sp}}\right\rangle\right)$, the hollowness parameters $\left(\left\langle\widetilde{D}_{e}\right\rangle\right.$ and $\left.\left\langle\widetilde{D}_{i}\right\rangle\right)$ and the ion Mach number $\left(\left\langle M_{A}\left(=v_{R} / v_{A}\right)\right\rangle\right)$, are shown in Table II, where $\langle\cdots\rangle$ means the time average after the relaxation $\left(\omega_{c i} t>5 \pi\right), v_{R}$ is the ion flow velocity at field-null line, and $v_{A}$ is the Alfvén velocity estimated by the density at field-null line and the magnetic field at the wall. In the zero $E(0)$ case, when the initial current profile is peaked in $\bar{s}=2,3$ (C3 and C5), both the ion and electron current profiles keep peaked $\left(\left\langle\widetilde{D}_{i}\right\rangle<0,\left\langle\widetilde{D}_{e}\right\rangle\right.$ $<0)$, while in the other cases $(\mathrm{C} 1, \mathrm{C} 2, \mathrm{C} 4$, and $\mathrm{C} 6)$ the ion current profile becomes peaked $\left(\left\langle\widetilde{D}_{i}\right\rangle<0\right)$ and the electron current profile becomes hollow $\left(\left\langle\widetilde{D}_{e}\right\rangle>0\right)$. On the other hand, the electron current profile keep its initial profile in each case of the finite $E(0)$. $\left\langle\widetilde{D}_{i}\right\rangle$ cannot be calculated because $J_{\phi, i}$ is too small.

Table II also shows the growth rate $\gamma_{n=1}$ of the $n=1$ mode for the ion average flow velocity $v_{z, i}$ in $z$ direction, where $n$ is the toroidal mode number. These growth rates are normalized by those obtained from the MHD simulation $\gamma_{\mathrm{MHD}}$. In all cases, $\gamma_{n=1}$ reduces to roughly $15 \%$ of $\gamma_{\mathrm{MHD}}$ because of the kinetic effect. In cases when the hollow current profile is realized after relaxation $(\mathrm{C} 1, \mathrm{C} 2, \mathrm{C} 4, \mathrm{C} 6, \mathrm{E} 2$, E4, and E6) $\gamma_{n=1}$ is smaller compared with the moderate kinetic case of the peaked current profile (C3, C5, E1, E3, and E5).

Though $\gamma_{n=1}$ in the kinetic equilibrium becomes surely smaller than that in MHD case, $\gamma_{n=1}$ remains finite. All of the modes take place because the full simulation are performed in this paper. We need to examine what mechanism causes the $n=1$ toroidal mode to grow. Let us pay attention to the fact that the $n=1$ mode consists of two physical modes, that is, the tilt mode and the shift mode. Figure 13 shows the schematic illustration of the flow pattern for the tilt mode and shift mode. The flow pattern of the tilt mode is

TABLE II. The time averaged parameters from the 2D simulation results, the ratio of field-null to vessel radius $\langle R\rangle / r_{D}$; the ratio of separatrix to vessel radius $\left\langle r_{\mathrm{sp}}\right\rangle / r_{D}$; the elongation $\left\langle E\left(=z_{\mathrm{sp}} / r_{\mathrm{sp}}\right)\right\rangle$; the plasma beta value at the separatrix $\left\langle\beta_{\mathrm{sp}}\right\rangle$; the electron hollowness parameter $\left\langle\widetilde{D}_{e}\right\rangle$; the ion hollowness parameter $\left\langle\widetilde{D}_{i}\right\rangle$; the Mach number $\left\langle M_{A}\right\rangle$, and the growth rates $\gamma_{\text {tilt }}$ of the tilt instability from the 3D simulation results. $\langle\cdots\rangle$ means the time average after the relaxation $\left(\omega_{c i} t>5 \pi\right)$, and $\gamma_{\mathrm{MHD}}$ is the tilt growth rate in the MHD simulation. The cases from C1 to C6 is the results from the initial condition of the zero $E(0)$ case, and the cases from E1 to E6 is the results from the initial condition of the finite $E(0)$ case.

\begin{tabular}{|c|c|c|c|c|c|c|c|c|c|c|c|}
\hline Case & $\bar{s}$ & $D$ & $\langle R\rangle / r_{D}$ & $\left\langle r_{\mathrm{sp}}\right\rangle / r_{D}$ & $\langle E\rangle$ & $\left\langle\beta_{\mathrm{sp}}\right\rangle$ & $\left\langle\widetilde{D}_{e}\right\rangle$ & $\left\langle\widetilde{D}_{i}\right\rangle$ & $\left\langle M_{A}\right\rangle$ & $\gamma_{n=1} / \gamma_{\mathrm{MHD}}$ & $\gamma_{\text {tilt }} / \gamma_{\mathrm{MHD}}$ \\
\hline $\mathrm{C} 1$ & 1 & -0.6 & 0.511 & 0.711 & 2.17 & 0.288 & 0.346 & -3.09 & 0.477 & $0.143 \pm 0.073$ & $0.051 \pm 0.059$ \\
\hline $\mathrm{C} 2$ & 1 & 0.2 & 0.512 & 0.709 & 2.66 & 0.333 & 0.810 & -1.58 & 0.390 & $0.112 \pm 0.054$ & $0.061 \pm 0.147$ \\
\hline $\mathrm{C} 3$ & 2 & -0.6 & 0.493 & 0.697 & 2.09 & 0.226 & -0.142 & -2.00 & 0.226 & $0.270 \pm 0.070$ & $0.295 \pm 0.078$ \\
\hline $\mathrm{C} 4$ & 2 & 0.2 & 0.492 & 0.696 & 2.69 & 0.247 & 0.535 & -0.865 & 0.169 & $0.181 \pm 0.037$ & $0.131 \pm 0.057$ \\
\hline $\mathrm{C} 5$ & 3 & -0.6 & 0.492 & 0.694 & 2.11 & 0.212 & -0.501 & -1.58 & 0.146 & $0.243 \pm 0.084$ & $0.288 \pm 0.032$ \\
\hline C6 & 3 & 0.2 & 0.489 & 0.693 & 2.71 & 0.237 & 0.101 & -0.582 & 0.101 & $0.055 \pm 0.074$ & $0.065 \pm 0.059$ \\
\hline E1 & 1 & -0.6 & 0.490 & 0.693 & 2.11 & 0.220 & -0.997 & & 0.006 & $0.285 \pm 0.022$ & $0.286 \pm 0.021$ \\
\hline E2 & 1 & 0.2 & 0.492 & 0.694 & 2.74 & 0.239 & 0.013 & & 0.004 & $0.168 \pm 0.020$ & $0.205 \pm 0.041$ \\
\hline E3 & 2 & -0.6 & 0.490 & 0.693 & 2.10 & 0.210 & -0.932 & & 0.009 & $0.247 \pm 0.059$ & $0.267 \pm 0.078$ \\
\hline E4 & 2 & 0.2 & 0.490 & 0.693 & 2.68 & 0.231 & 0.068 & & 0.008 & $0.130 \pm 0.066$ & $0.173 \pm 0.052$ \\
\hline E5 & 3 & -0.6 & 0.490 & 0.693 & 2.14 & 0.207 & -0.922 & & 0.009 & $0.319 \pm 0.027$ & $0.327 \pm 0.032$ \\
\hline E6 & 3 & 0.2 & 0.492 & 0.694 & 2.67 & 0.233 & 0.070 & & 0.002 & $0.104 \pm 0.062$ & $0.115 \pm 0.060$ \\
\hline
\end{tabular}




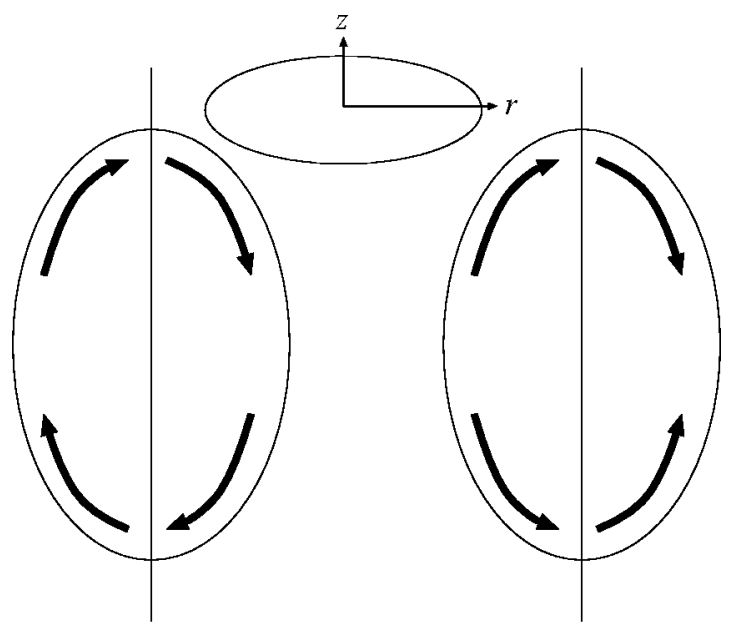

(a) tilt mode

(b) shift mode

FIG. 13. Schematic illustration of (a) the tilt mode and (b) the shift mode. The arrow shows the average flow.

the rotation around the axis normal to the $z$ axis, while the flow pattern which makes the central axis of the FRC plasma move radially appears in the shift mode. So we separate the $n=1$ mode into the tilt and shift modes by assuming that the flow is symmetric in the tilt mode and anti-symmetric in the shift mode about an equatorial plane. If we define the next four values

$$
\begin{aligned}
& v_{r}^{-}=v_{r}(z)-v_{r}(-z), \\
& v_{z}^{+}=v_{z}(z)+v_{z}(-z), \\
& v_{r}^{+}=v_{r}(z)+v_{r}(-z), \\
& v_{z}^{-}=v_{z}(z)-v_{z}(-z),
\end{aligned}
$$

the flow $\left(v_{r}^{-}, v_{z}^{+}\right)$corresponds to the tilt flow, while the flow $\left(v_{r}^{+}, v_{z}^{-}\right)$corresponds to the shift flow. Figure 14 shows the time history of the mode amplitudes $\Delta v_{z}^{+}$and $\Delta v_{z}^{-}$for $v_{z}^{+}$ and $v_{z}^{-}$, respectively, in the cases of (a) C2 and (b) C5. In the case of $\mathrm{C} 2$, both $\Delta v_{z}^{+}$and $\Delta v_{z}^{-}$increase until $t / t_{\mathrm{A}}=3$, where $t_{\mathrm{A}}$ is the Alfvén transit time. After this period $\Delta v_{z}^{+}$ decreases while $\Delta v_{z}^{-}$continues to increase $\left(\Delta v_{z}^{+}<\Delta v_{z}^{-}\right)$. These phenomena indicate that the shift mode dominantly continues to grow and the tilt mode damps, that is to say, is stabilized from $t / t_{\mathrm{A}}=3$. In the case of C5, on the other hand, both $\Delta v_{z}^{+}$and $\Delta v_{z}^{-}$increase, that is, both the tilt and shift modes grow up. However, the tilt mode is dominant in the late phase because $\Delta v_{z}^{+}$is always larger than $\Delta v_{z}^{-}$. Let us examine directly the flow pattern to make clear which mode is dominant in each case, the tilt or shift mode. Figure 15 shows the ion flow pattern of the $n=1$ mode, the tilt component $\left(v_{r}^{-}, v_{z}^{+}\right)$and the shift component $\left(v_{r}^{+}, v_{z}^{-}\right)$on $r-z$ plane at $t / t_{\mathrm{A}}=5$. It is obviously shown in Fig. 15 that the shift mode is dominant in the case of $\mathrm{C} 2$ and the tilt and shift modes grow in the case of C5. This shift mode is generated on the inside of the plasma and the plasma oscillates around the $z$ axis. We also perform the simulation in which the shift mode is forced not to be excited. From this calculation, we
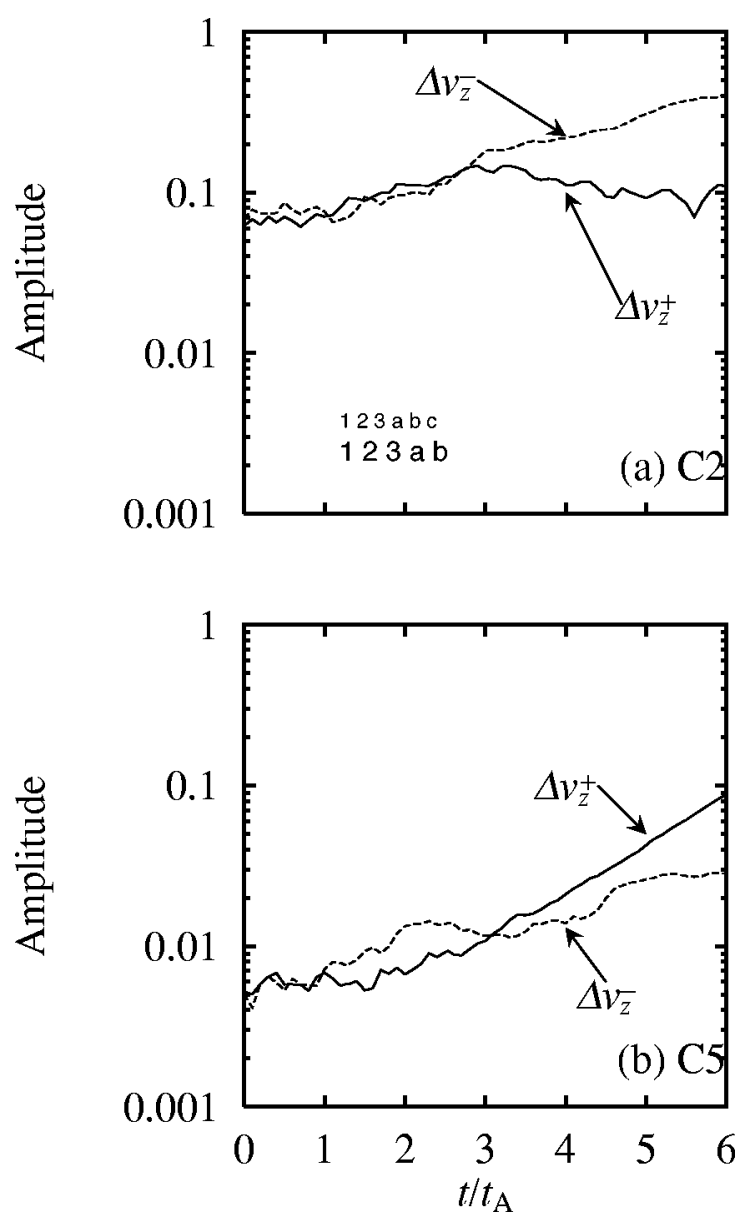

FIG. 14. Time history of the mode amplitudes $\Delta v_{z}^{+}$(solid line) and $\Delta v_{z}^{-}$ (dashed line) for (a) C2 and (b) C5.

find that the shift mode does not affect on the tilt mode. The appearance of the shift mode does not matter in the investigation of the tilt instability.

We calculate the growth rate of the tilt mode $\gamma_{\text {tilt }}$ from the time history of the $\Delta v_{z}^{+}$(Table II). In cases when the hollow current profile is realized after the relaxation $(\mathrm{C} 1, \mathrm{C} 2$, C4, C6, E2, E4, and E6), $\gamma_{\mathrm{tilt}} / \gamma_{\mathrm{MHD}}$ is smaller compared with the case of the peaked current profile $(\mathrm{C} 3, \mathrm{C} 5, \mathrm{E} 1, \mathrm{E} 3$, and E5).

There are several parameters which are related to the stabilization of the tilt mode. For instance, the plasma beta value $\beta$, the hollowness parameter $D$, the Alfvén Mach number $M_{A}$, and the finite Larmor radius parameter $\bar{s}$. We discuss the relationship between the growth rate and three parameters here.

Figure 16(a) shows the dependence of the tilt growth rate $\gamma_{\text {tilt }}$ on the plasma beta value $\left\langle\beta_{\mathrm{sp}}\right\rangle$ at the separatrix. The tilt growth rate tends to decrease as $\left\langle\beta_{\mathrm{sp}}\right\rangle$ increases in the same way as the result by Nishimura et al. ${ }^{11}$ These results show that the separatrix beta value is relevant to the tilt stabilization, and that the anchoring ions can play an important role in the tilt stabilization.

Figure 16(b) shows the dependence of the tilt growth rate $\gamma_{\text {tilt }}$ on the electron hollowness parameter $\left\langle\widetilde{D}_{e}\right\rangle$. It is worthy of notice that the tilt growth rate is remarkably re- 


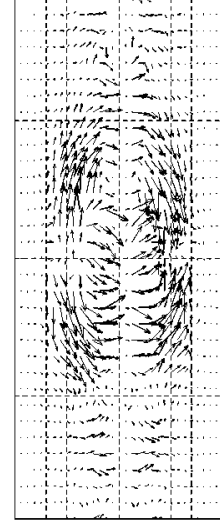

(a) $n=1(C 2)$

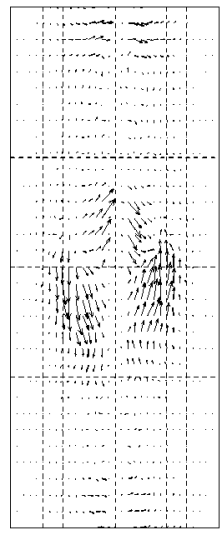

(d) $n=1(C 5)$

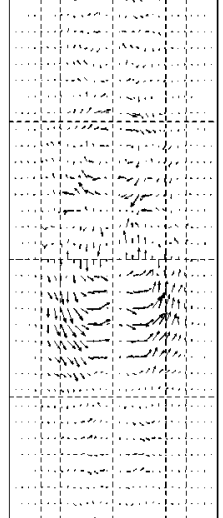

(b) tilt (C2)

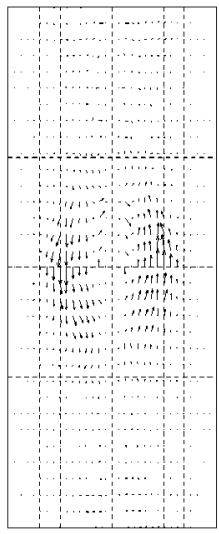

(e) tilt (C5)

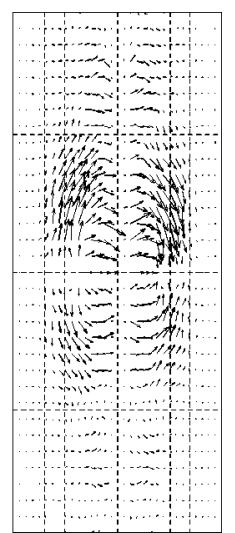

(c) shift (C2)

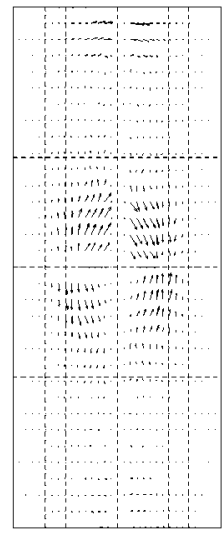

(f) shift (C5)
FIG. 15. The ion flow pattern of the $n=1$ mode, the tilt component $\left(v_{r}^{-}, v_{z}^{+}\right)$, and the shift component $\left(v_{z}^{+}, v_{z}^{-}\right)$in $r-z$ plane for (a)-(c) C2 and (d) - (f) $\mathrm{C} 5$ at $\omega_{c i} t=5 \pi$.

duced when the electron current profile is hollow $\left(\left\langle\widetilde{D}_{e}\right\rangle\right.$ $>0)$. These results indicate that the electron hollowness parameter has much to do with the tilt stabilization. Since Fig. 16(b) demonstrates all sorts of the tilt growth rates obtained from various initial conditions, such as the zero $E(0)$ and finite $E(0)$ cases, it is important to point out that there is a clear correlation between the growth rate $\gamma_{\text {tilt }}$ and the electron hollowness parameter $D_{e}$. This tendency coincides with the analysis of the experiments by Steinhauer and Ishida. ${ }^{20}$

Figure 16(c) indicates the dependence of the tilt growth rate $\gamma_{\text {tilt }}$ on the Mach number $\left\langle M_{A}\right\rangle$ of the ion toroidal flow, where $M_{A}$ is the ratio of the ion toroidal flow velocity at the field-null line to the Alfvén velocity. In the MHD simulation, ${ }^{2}$ the tilt mode is stabilized due to the spin stabilization effect when $M_{A}>1$. In this particle simulation, on the other hand, the tilt stabilization becomes visible in the region of $M_{A} \approx 0.5$. Therefore, this stabilization is not explained directly by the spin stabilization effect. In Fig. 16(c), the tilt growth rate $\gamma_{\text {tilt }}$ tends to reduce when $\bar{s}$ decreases and $M_{A}$ increases. These results suggest that the ion toroidal motion partially contributes to the tilt stabilization.

These results lead us to the conclusion that the plasma confinement is broken by the tilt instability in the moderate kinetic case of the peaked current profile, while the tilt mode
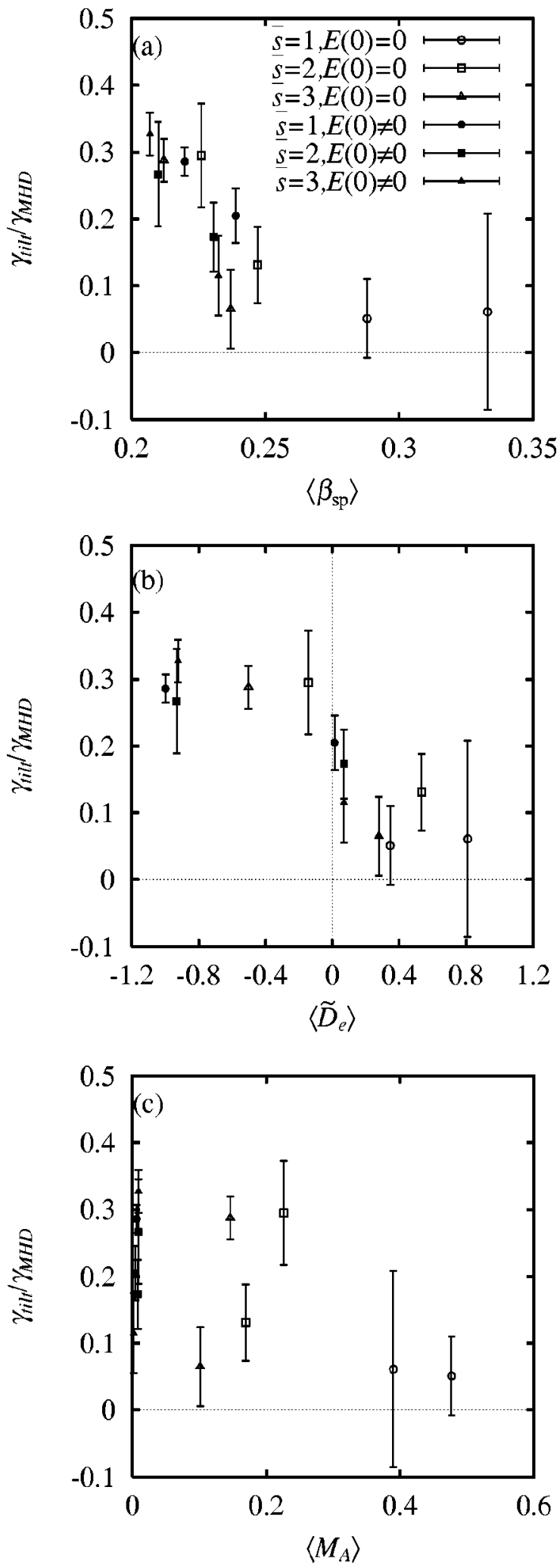

FIG. 16. The dependence of the growth rate $\gamma_{\text {tilt }} / \gamma_{\mathrm{MHD}}$ of the tilt instability on (a) the plasma beta value $\left\langle\beta_{\mathrm{sp}}\right\rangle$ at the separatrix on the midplane, (b) the electron hollowness parameter $\left\langle\widetilde{D}_{e}\right\rangle$, and (c) the Mach number $\left\langle M_{A}\right\rangle$ of the ion toroidal flow at the field field-null line. The open circle shows the case of $\bar{s}=1$, the open square shows the case of $\bar{s}=2$, and the open triangle shows the case of $\bar{s}=3$ under the initial condition of the zero $E(0)$ case, respectively. The closed circle shows the case of $\bar{s}=1$, the closed square shows the case of $\bar{s}=2$, and the closed triangle shows the case of $\bar{s}=3$ under the initial condition of the finite $E(0)$ case, respectively. 
tends to be stabilized and the shift mode is dominant in the cases of the hollow current profile and high separatrix beta.

\section{SUMMARY}

We perform the two-dimensional electromagnetic particle simulation to investigate the profile relaxation from an MHD equilibrium to a kinetic one and to clarify the property of the kinetic equilibrium of the field-reversed configurations independently of the tilt instability. And then we perform the three-dimensional full electromagnetic particle simulation using the kinetic profile obtained from the two-dimensional simulation as the initial condition to examine the stability of the kinetic equilibrium against the tilt mode. Our results summarized as follows.

\section{A. Profile relaxation from MHD to kinetic}

The relaxation oscillation takes place when the profile relaxes from an MHD equilibrium to a kinetic one. In the full kinetic case when the relaxed state is far from the MHD equilibrium, the large relaxation oscillation is excited. The relaxation oscillation with the frequency $\omega \approx 2 \omega_{c i}$ is gradually damped in $\omega_{c i} t \approx 5 \pi$. Near the field-null line, the hollow profile of the electron current is realized because of the gradient-B drift. The ion current profile becomes peaked through the ion meandering orbit effect. Namely, the effect of the particle orbit takes an important role in the selfgeneration of the kinetic current profile near the field-null line. The ion finite Larmor radius effect generates the radial electric field in the narrow periphery region near the separatrix. Because the generated $\mathrm{E} \times \mathrm{B}$ drift has the same sign as the electron diamagnetic drift, the electron current density increases in the periphery and then electron profile tends to be more hollow. On the other hand, the generated $\mathrm{E} \times \mathrm{B}$ drift has an opposite sign as the ion diamagnetic drift. Since the region of the strong electric field is smaller than the ion Larmor radius, however, the electric field acts on ions less effectively. In this way, the electron hollow current profile and the ion peaked one are spontaneously realized after the relaxation from the MHD equilibrium to the kinetic one.

\section{B. The stability against the tilt mode}

We analyze the stability against the tilt mode by separating the $n=1$ mode into the tilt and shift modes. The growth rate of the tilt instability in all cases reduces to a small value because of the kinetic effect. In the system where the hollow current profile is realized after the profile relaxation, the growth of the tilt instability is suppressed and the shift mode is dominant, while in the system with peaked current profile, the tilt instability grows. From the investigation into the relationship between the tilt growth rate and several parameters, we find that the electron hollowness parameter and the separatrix beta value are important keys to solving the problem of the tilt stabilization. We will discuss the physical mechanism of the reduction of the tilt growth rate and the tilt stabilization due to the kinetic and profile effects in the next paper.

\section{ACKNOWLEDGMENTS}

This simulation is performed on the MISSION System (Grand Man-Machine Interactive System for Simulation) at the National Institute for Fusion Science. This work is supported by a Grant-in-Aid from the Ministry of Education, Culture, Sports, Science and Technology in Japan (No. 13640414).

${ }^{1}$ R. A. Clemente and J. L. Milovich, Phys. Lett. A 85, 148 (1981).

${ }^{2}$ R. Horiuchi and T. Sato, Phys. Fluids B 1, 581 (1989).

${ }^{3}$ J. T. Slough, A. L. Hoffman, R. D. Milroy, R. Maqueda, and L. C. Steinhauer, Phys. Plasmas 2, 2286 (1995).

${ }^{4}$ J. C. E. Seyler and D. C. Barnes, Phys. Fluids 24, 1989 (1981).

${ }^{5}$ J. L. Schwarzmeier and C. E. Seyler, Phys. Fluids 27, 2151 (1984).

${ }^{6}$ H. R. Lewis, D. C. Barnes, J. L. Schwarzmeier, and C. E. Seyler, Phys. Fluids 28, 3546 (1985).

${ }^{7}$ D. C. Barnes, J. L. Schwarzmeier, H. R. Lewis, and C. E. Seyler, Phys. Fluids 29, 2616 (1986).

${ }^{8}$ R. D. Milroy, D. C. Barnes, R. C. Bishop, and R. B. Webster, Phys. Fluids B 1, 1225 (1989).

${ }^{9}$ R. Horiuchi and T. Sato, Phys. Fluids B 2, 2652 (1990).

${ }^{10}$ J. W. Cobb, T. Tajima, and D. C. Barnes, Phys. Fluids B 5, 3227 (1993).

${ }^{11}$ K. Nishimura, R. Horiuchi, and T. Sato, Phys. Plasmas 4, 4035 (1997).

${ }^{12}$ R. Horiuchi, K. Nishimura, T. H. Watanabe, and T. Sato, Nucl. Fusion 39, 2083 (1999).

${ }^{13}$ K. Nishimura, R. Horiuchi, and T. Sato, Phys. Plasmas 6, 3459 (1999).

${ }^{14}$ H. Ohtani, R. Horiuchi, T. Sato, and H. Kawabuchi, J. Plasma Fusion Res. (to be published).

${ }^{15}$ E. V. Belova, S. C. Jardin, H. Ji, M. Yamada, and R. Kulsrud, Phys. Plasmas 7, 4996 (2000)

${ }^{16}$ E. V. Belova, S. C. Jardin, H. Ji, M. Yamada, and R. Kulsrud, Phys. Plasmas 8, 1267 (2001).

${ }^{17}$ D. C. Barnes, Phys. Plasmas 8, 4856 (2001).

${ }^{18}$ D. C. Barnes, Phys. Plasmas 8, 4864 (2001).

${ }^{19}$ D. C. Barnes, Phys. Plasmas 9, 560 (2002).

${ }^{20}$ L. C. Steinhauer and A. Ishida, Phys. Fluids B 4, 645 (1992).

${ }^{21}$ L. C. Steinhauer, A. Ishida, and R. Kanno, Phys. Plasmas 1, 1523 (1994).

${ }^{22}$ R. Kanno, A. Ishida, and L. C. Steinhauer, J. Phys. Soc. Jpn. 64, 463 (1995).

${ }^{23}$ M. Tanaka and T. Sato, Phys. Fluids 29, 3823 (1986).

${ }^{24}$ C. K. Birdsall and A. B. Langdon, Plasma Physics Via Computer Simulation (McGraw-Hill, New York, 1985).

${ }^{25}$ R. Horiuchi and T. Sato, Phys. Plasmas 1, 3587 (1994).

${ }^{26}$ R. Courant, K. O. Friedriches, and H. Lewvy, Math. Ann. 100, 32 (1928). 